\title{
Folate Receptor-Targeting Gold Nanoclusters as Fluorescence Enzyme Mimetic Nanoprobes for Tumor Molecular Colocalization Diagnosis
}

Dehong $\mathrm{Hu}^{*}$, Zonghai Sheng ${ }^{*}$, Shengtao Fang, Yanan Wang, Duyang Gao, Pengfei Zhang, Ping Gong, Yifan $\mathrm{Ma}$, and Lintao Cai ${ }^{\bowtie}$

Guangdong Key Laboratory of Nanomedicine, CAS Key Laboratory of Health Informatics, Shenzhen Institutes of Advanced Technology, Chinese Academy of Sciences, Shenzhen, 518055, China

* Dehong Hu and Zonghai Sheng equally contributed to this work.

$\triangle$ Corresponding author: Professor Lintao Cai, Shenzhen Institutes of Advance Technology, Chinese Academy of Science, 1068 Xueyuan Avenue, Shenzhen University Town, Shenzhen, P. R. China 518055. Tel: +86-755-86392210; Fax: +86-755-86392299; Email address: lt.cai@siat.ac.cn

(c) Ivyspring International Publisher. This is an open-access article distributed under the terms of the Creative Commons License (http://creativecommons.org/ licenses/by-nc-nd/3.0/). Reproduction is permitted for personal, noncommercial use, provided that the article is in whole, unmodified, and properly cited.

Received: 2013.07.26; Accepted: 2013.09.27; Published: 2014.01.02

\begin{abstract}
Nanoprobes with enzyme-like properties attracted a growing interest in early screening and diagnosis of cancer. To achieve high accuracy and specificity of tumor detection, the design and preparation of enzyme mimetic nanoprobes with high enzyme activity, tumor targeting and excellent luminescence property is highly desirable. Herein, we described a novel kind of fluorescence enzyme mimetic nanoprobe based on folate receptor-targeting Au nanoclusters. The nanoprobes exhibited excellent stability, low cytotoxicity, high fluorescence and enzyme activity. We demonstrated that the nanoprobes could be used for tumor tissues fluorescence/visualizing detection. For the same tumor tissue slice, the nanoprobes peroxidase staining and fluorescent staining were obtained simultaneously, and the results were mutually complementary. Therefore, the fluorescence enzyme mimetic nanoprobes could provide a molecular colocalization diagnosis strategy, efficiently avoid false-positive and false-negative results, and further improve the accuracy and specificity of cancer diagnoses. By examining different clinical samples, we demonstrated that the nanoprobes could distinguish efficiently cancerous cells from normal cells, and exhibit a clinical potential for cancer diagnosis.
\end{abstract}

Key words: Gold Nanoclusters, Fluorescence, Enzyme Mimetic, Tumor Diagnosis, Molecular Colocalization

\section{Introduction}

Cancer is considered a worldwide mortal sickness and has become a major public concern. Identification and detection of cancer have especially importance in the survival of patients with cancer. Thus, it is highly desirable to develop rapid, sensitive, and specific methods to diagnose cancers. Currently, typical methods have been applied in the field of cancer diagnosis in vitro and in vivo. [1-5] Medical imaging in vivo plays an important role in the preoperative diagnosis of cancer.[6-11] Optical imaging as a novel imaging modality would have more wider applications in biomedical applications because of its advantages such as low cost, low-energy radiation, high sensitivity, real-time monitoring, non-invasive or minimally invasive testing.[12] Furthermore, near infrared (NIR) fluorescence imaging can be used for 
the diagnosis in deep tissues and organs owning to its high sensitivity and penetration depth in biological tissues.[13] Recent advances in nanotechnology have provided platforms for developing nanoscale fluorescent nanoprobes, for example, quantum dots (QDs). [14] Although compared to traditional fluorescent nanoprobes, QDs have stronger fluorescent intensity and more durable signal. Its used in living cells or animals is limited due to its intrinsic toxicity, which may cause risks to human subjects.[15,16] In contrast to QDs, the low toxicity and ultrafine size of nanomaterials are highly attractive for tumor imaging and therapy. Molecular clusters of pure metals, especially gold, due to their inert reactivity, have great potential for application in biomedicine.

Gold nanoclusters (Au NCs), typically consisting of several to tens of atoms, are highly attractive for tumor imaging due to their ultrasmall size, good biocompatibility, excellent luminescence properties and many potential applications.[17-19] In particular, highly fluorescent $\mathrm{Au}$ NCs have been successfully prepared using bovine serum albumin (BSA) as a template. $[18,20]$ A large number of studies have recently focused on application of NIR fluorescent BSA-stabilized Au NCs for the detection of various analytes,[20,21] and for NIR biomedical imaging of cancer in vitro and in vivo.[22,23] However, the veracity of these methods is not satisfactory. To overcome this problem, pathological diagnosis could be employed as a supplementary diagnosis.

Pathological diagnosis has been the gold standard for diagnosis in tumor and its role has also included the tumor differentiation, the degree of morphological changes, the elucidation of etiology, pathogenesis, clinicopathological correlation, and prognostication.[24, 25] During the last decades, the development of immunohistochemistry (IHC) has provided a basis for the molecular characterization of tumors using antibodies to localize molecules expressed by tumor cells, and it has been developed to be a powerful tool for both research and diagnostic purposes.[26-28] Horseradish peroxidase (HRP) is the most common used enzyme in IHC. But, one of the primary problems associated with the use of HRP is nonspecific staining (false-positives) that result from endogenous peroxide activity in certain tissues. A second drawback is inadequate fixation that can cause weak or false negative staining (false-negative), because there is no optimal standard fixation time for every antigen. Moreover, the sensitivity of HRP is easy to be affected by microorganisms as well as the antibacterial agents.

Recently, nanoparticles (NPs) have received increasing attention in enzyme mimetic research due to several distinct properties, such as narrow size dis- tribution, high surface area to volume ratio, and abundance of reactive groups for further functionalization.[29-32] In 2007, Yan and coworkers reported that $\mathrm{Fe}_{3} \mathrm{O}_{4}$ NPs possessed intrinsic enzyme activity similar to that found in natural HRP.[29] Their research paved the way for NPs as enzyme mimetics.[33-37] In contrast to natural enzyme, the preparation, purification and storage of the enzyme mimetic are usually time-saving and inexpensive. Furthermore, the enzyme mimetics can overcome the drawbacks of nature enzymes, such as sensitivity of catalytic activity to environmental conditions, low stability due to denaturation and digestion. Recently, Yan and coworkers further used iron oxide NPs as HRP mimetics to detect tumor tissues in pathological diagnosis, and resolve the low sensitivity and degradation characteristics of HRP.[38] Nevertheless, substantial efforts are still needed for resolving the false-positives and false-negative in IHC.

More recently, it was reported that BSA-stabilized Au NCs could possess highly enzyme mimetic activity, and which will be highly beneficial for a wide range of applications in clinical diagnostics.[39,40] In this study, we integrated the feature of BSA stabilized Au NCs with NIR fluorescent imaging and peroxidase mimetics activity to develop a rapid, accurate, low-cost and universal method for tumor tissues fluorescence/visualizing diagnosis. These extended previous our studies involving hybrid gold-gadolinium NCs for triple-modal imaging of tumor diagnosis.[41] Folic acid (FA) was immobilized on the surface of Au NCs to target and visualize tumor tissues that overexpress folate receptor (FR). By using a one-step incubation of our NCs-FA fluorescent peroxidase mimetics nanoprobes-based colocalization staining method, the tumor tissues could be visualized based on microscopic imaging with bright field (the oxidation of peroxidase substrates) and fluorescent images in the same section, simultaneously, and the two images were mutually complementary. The method could avoid false-positive and false-negative in histochemical diagnostic of tumor. A series of subsequent investigations revealed the NCs-FA fluorescent peroxidase mimetics nanoprobes could distinguish cancerous cells from normal cells. Such well-defined nanocluster-fluorescent peroxidase mimetics staining has the potential to become a molecular diagnostic tool for cancer imaging and prediction of cell cancerization.

\section{Methods}

\section{Materials}

$\mathrm{HAuCl}_{4} .4 \mathrm{H}_{2} \mathrm{O}$, bovine serum albumin $(66000$ Da), goat anti mouse IgG-Alexa Fluor 568, goat anti 
mouse IgG-HRP and FA were purchased from Sigma Aldrich; mouse anti-FR protein antibody and mouse anti-beta actin antibody were purchased from Abcam; o-Phenylenediamine (OPD, 99.5\%), 3,3',5,5'-Tetramethylbenzidine (TMB, 99\%), 3,3'-Diaminobenzidine (DAB, 99\%), 5-Aminosalicylic acid (5-ASA, 98.5\%) and 3-Amino-9-ethylcarbazole (AEC) were purchased from J\&KCHEMICA without further purification; Hydrogen peroxide $(30 \%)$, hydrochloric acid $(\mathrm{HCl}$, $37 \%)$, nitric acid $\left(\mathrm{HNO}_{3}, 65 \%\right)$, sodium hydroxide $(\mathrm{NaOH}, 99.99 \%)$ and ethanol were obtained from Sinopharm Chemical Reagent Co., Ltd (Shanghai, China); phosphate-buffered saline (PBS, pH 7.4), fetal bovine serum (FBS) and Dulbecco's Modified Eagle's Medium (DMEM) were purchased from Gibco Life Technologies (AG, Switzerland). Clinical tumor and normal tissues were obtained from the tissue bank of the second people's hospital of Shenzhen (Shenzhen). All other chemicals used in this study were of analytical reagent grade and used without further purification. Superpure water $\left(18.25 \mathrm{M} \Omega . \mathrm{cm}, 25{ }^{\circ} \mathrm{C}\right)$ was used to prepare all solutions. BALB/c athymic nude mice and BALB/C mice were maintained under aseptic conditions in a small animal isolator. All food, water, bedding and cages were autoclaved before use.

\section{Synthesis of Au NCs}

$\mathrm{Au}$ NCs with NIR emissions prepared according to our previous report.[20] All glassware was washed with aqua regia $\left(\mathrm{HCl}: \mathrm{HNO}_{3}\right.$ volume ratio 3: 1), and rinsed with ethanol and superpure water, respectively. In a typical experiment, $\mathrm{HAuCl}_{4} 4 \mathrm{H}_{2} \mathrm{O}$ aqueous solution $\left(25 \mathrm{~mL}, 10 \mathrm{mM}, 37{ }^{\circ} \mathrm{C}\right)$ was added to BSA solution $\left(25 \mathrm{~mL}, 50 \mathrm{mg} \mathrm{mL}^{-1}, 37{ }^{\circ} \mathrm{C}\right)$ under vigorous stirring. $\mathrm{NaOH}$ solution $(0.5 \mathrm{~mL}, 1 \mathrm{M})$ was introduced $2 \mathrm{~min}$ later, and the reaction was allowed to proceed under vigorous stirring at $37{ }^{\circ} \mathrm{C}$. The color of the solution changed from light yellow to light brown, and then to deep brown. The reaction was completed in 12 $\mathrm{h}$, and the solution was stored at $4{ }^{\circ} \mathrm{C}$. The as-prepared $\mathrm{Au}$ NCs have relatively high photoluminescence quantum yield $(\sim 6 \%)$.

\section{Synthesis of the NCs-FA nanoprobes}

FA was conjugated to amino-groups on the surface of Au NCs to form the NCs-FA nanoprobes. First, FA was reacted with EDC/NHS (molar ratio of FA: EDC: NHS is 1: 1.5: 2) in anhydrous dimethyl sulfoxide (DMSO, $2.5 \mathrm{~mL}$ ) and the mixture was stirred in the dark for $12 \mathrm{~h}$ at $50{ }^{\circ} \mathrm{C}$. The residue was removed by filtration under reduced pressure and centrifugation (16 $000 \mathrm{rpm}$ ) for $10 \mathrm{~min}$, and the activated FA was extracted with by adding $10 \mathrm{~mL}$ anhydrous acetone. Second, the activated FA was dissolved in Tris buffer $(1 \mathrm{~mL}, 10 \mathrm{mM}, \mathrm{pH} 8.0)$ and the Au NCs were adjusted to a concentration of $10 \mathrm{mM}$ in $2 \mathrm{~mL}$ Tris buffer. The two solutions were then mixed and stirred in the dark for $12 \mathrm{~h}$ at $4{ }^{\circ} \mathrm{C}$.

\section{Purification}

Purification of the NCs-FA nanoprobes was performed by ultra centrifugation using filter devices (Millipore, 10kD) at $5000 \mathrm{rpm}$. This step was repeated several times, by filling the tubes with PBS buffer and centrifuging again, until the desired purification rate was reached. Then the solution was filtered through a $0.2 \mu \mathrm{m}$ membrane to remove the largest impurities. The resulting product was stored at $-20^{\circ} \mathrm{C}$.

\section{Spectra assay}

UV-Vis absorption spectra were obtained with a PerkinElmer Lambda 25 UV-Vis spectrophotometer. The fluorescent spectra were obtained by a fluorescence spectrometer (F900, Edinburgh Instruments Ltd.).

\section{High resolution transmission electron microscopy (HRTEM) image}

HRTEM image was used to obtain detailed structural and morphological information about the samples and was carried out using a FEI Tecnai F20 HRTEM. The samples for HRTEM images were prepared by depositing a drop of a diluted colloidal solution on a carbon grid and allowing the liquid to dry in air at room temperature.

\section{Measurement of the relative enzyme activity of the NCs-FA nanoprobes and HRP}

A TMB solution was used to detect relative activity of the NCs-FA nanoprobes and HRP, respectively. Experiments were carried out using $1.0 \times 10^{-12} \mathrm{M}$ NCs-FA nanoprobes or $1.0 \times 10^{-11} \mathrm{M}$ HRP in a reaction volume of $1 \mathrm{~mL}$, in $0.1 \mathrm{M}$ PBS buffer, with $500 \mu \mathrm{M}$ TMB as substrate. The $\mathrm{H}_{2} \mathrm{O}_{2}$ concentration was 300 $\mathrm{mM}$ for NCs-FA nanoprobes and $10 \mathrm{mM}$ for HRP. All test conditions was at $35^{\circ} \mathrm{C}$ in a pH 4.0 PBS (0.1M) buffer. After $5 \mathrm{~min}$, the oxidation reaction was stopped by the addition of an equal volume of $0.1 \mathrm{M}$ sulfuric acid. The relative activity was defined as follows:

The relative activity $(\%)=\mathrm{A}_{1} / \mathrm{A}_{2} \times 100 \%$

Where $A_{2}$ was the maximum absorbance, $A_{1}$ was the sample absorbance measured at the same conditions.

\section{Cell culture}

The cell lines SKOV3 (human evarian cancer), MCF-7 cells (human breast cancer cells), HepG2 (human liver cancer), MDA-MB-231 (human breast cancer), H460 (human lung cancer) and 293T (human embryonic kidney) were cultured in Dulbecco's 
Modified Eagle Medium (DMEM, HyClone) supplemented with $10 \%(\mathrm{v} / \mathrm{v})$ fetal bovine serum, $1 \%(\mathrm{v} / \mathrm{v})$ penicillin, and $1 \%(\mathrm{v} / \mathrm{v})$ streptomycin. Cells were incubated in a humidified incubator at $37{ }^{\circ} \mathrm{C}$ with $5 \%$ $\mathrm{CO}_{2}$.

\section{Cell viability assay}

MCF-7 (HepG2, 293T) cells were planted into 96-well plates for the cell viability methyl thiazolyl tetrazoliym (MTT) assay. All cells in logarithmic growth were treated with the NCs-FA nanoprobes at various concentrations from 3 to $100 \mu \mathrm{mol} \mathrm{mL}^{-1} \mathrm{com}-$ pared to the cells treated with PBS as control. After 24 $\mathrm{h}$, the cell viability was evaluated by the MTT assay. Each experiment was repeated at least five times to obtain the average value. The relative viability ratio of treated groups was calculated based on the intensity compared to controls.

\section{Assessments of FR expression level}

The five tumor cell lines (MCF-7, HepG2, SKOV3, MDA-MB-231 and H460) were used to evaluate receptor expression. FR expression was assessed by western blotting as previously described with modifications. Cell lysates samples of each type were run on a $10 \%$ SDS-polyacrylamide gel and transferred to a nitrocellulose membrane blocked with $5 \%$ non-fat milk, $0.1 \%$ Tween 20 in PBS for $30 \mathrm{~min}$, and then incubated overnight at $4{ }^{\circ} \mathrm{C}$ with a 1:2 000 dilution of anti-FR protein monoclonal antibody. The anti-FR protein monoclonal antibody was detected using a 1:6 000 dilution of goat anti-mouse IgG conjugated to HRP, and developed with Pierce ECL substrate.

\section{Confocal laser microscopy assay}

For in vitro studies, $1 \times 10^{5} \mathrm{MCF}-7$ and HepG2 cells were seeded on a glass-bottomed culture dish. After $24 \mathrm{~h}$, the NCs-FA nanoprobes (NCs) were incubated with different cells (MCF-7, HepG2) for $1 \mathrm{~h}$ at 37 ${ }^{\circ} \mathrm{C}$. To remove the unbound conjugates, the cells were washed three times for 10 min by using shaking incubation $(30 \mathrm{rpm})$ in Tris buffer and fixed with 3.7\% formaldehyde solution $(1 \mathrm{~mL})$ and incubated at shaking incubation (20 rpm) each for $20 \mathrm{~min}$. The nuclear dye Hoechst was used as a positive control to stain nuclei in the experiment. Images of cells were acquired using a Leica DMI6000 inverted microscope with a Leica TCS SP5 confocal scanning system.

\section{Animal model}

All animal experiments were conducted in agreement with the "Principles of Laboratory Animal Care" (NIH publication no. 86-23, revised 1985). The guidelines of the Institute for Nutritional Science of Chinese Academy of Sciences were also respected. Six-to-seven week old male BALB/c athymic nude mice and BALB/c mice were maintained under aseptic conditions in a small animal isolator and were housed in a group of five in standard cages with free access to food and water and a $12 \mathrm{~h}$ light/dark cycle. All animals acclimated to the animal facility for at least 7 days before experimentation. All possible parameters that may cause social stress, like group size, type (treated and nontreated), etc., among the experimental animals were carefully monitored and avoided. Animals were observed daily for any behavioral abnormalities and weighed weekly.

\section{In vivo NIR fluorescent imaging}

To perform in vivo NIR fluorescent imaging, male nude mice bearing MCF-7 tumors were intravenously injected with NCs-FA $(500 \mathrm{mg} / \mathrm{kg})$. The mouse was imaged using the Maestro in vivo imaging system (CRI, Inc., excitation, 435-480 nm; emission, $490 \mathrm{~nm}$ long-pass). The tunable filter was automatically stepped in $5 \mathrm{~nm}$ increments from 600 to $800 \mathrm{~nm}$, whereas the camera captured images at each wavelength interval with constant exposure. Spectral unmixing algorithms were applied to create unmixed images of NCs-FA.

\section{Histology}

Five healthy female Balb/c mice were injected with NCs-FA $(500 \mathrm{mg} / \mathrm{kg}, 30$ days). Other five healthy Balb/c mice were used as the untreated control. Major organs from those mice were harvested, fixed in $4 \%$ neutral buffered formalin. The excised organs were embedded in Tissue-Tek OTC compound (Sakura Finetechnical Co., Ltd., Tokyo, Japan) and frozen in a deep freezer at $-70{ }^{\circ} \mathrm{C}$. The tissue was sectioned into $10 \mu \mathrm{m}$ slices, stained with hematoxylin and eosin (H\&E) and examined by a digital microscope (Leica QWin). Examined tissues include heart, liver, spleen, lung, and kidney.

\section{Staining of tumor xenografts and clinical specimens}

Tissue sections were hydrated progressively using an ethanol gradient. Endogenous peroxidase activity was quenched by incubation with $0.3 \% \mathrm{H}_{2} \mathrm{O}_{2}$ in methanol for $30 \mathrm{~min}$. After rinsing, the tissue sections were boiled in $10 \mathrm{mM}$ citrate buffer $(\mathrm{pH} 6.0)$ at $100{ }^{\circ} \mathrm{C}$ for $30 \mathrm{~min}$, cooled to room temperature, blocked with $5 \%$ goat serum in PBS for $1 \mathrm{~h}$ at $37^{\circ} \mathrm{C}$, washed and then incubated with the NCs-FA nanoprobes (1.8 $\mathrm{mM}$ ) for $1 \mathrm{~h}$ at $37{ }^{\circ} \mathrm{C}$, and then rinsed in PBS. Freshly prepared DAB was added for color development. The nuclear dye Hoechst was used as a positive control to stain nuclei in the experiment. The stained sections were analysed under a microscope equipped with a Nuance multispectral imaging system (CRi, Woburn, MA). 
IHC staining of tissue sections by anti-FR Abs was performed to compare tumor-binding specificity and staining quality with the NCs-FA nanoprobes. Briefly, after blocking in serum, tissue sections were incubated at $4{ }^{\circ} \mathrm{C}$ overnight with a 1:300 dilution of mouse Anti-FR protein monoclonal antibody (Abcam). The bound antibody was detected by incubating the tissues with a 1:1,000 dilution of HRP-conjugated anti-mouse antibody at $37^{\circ} \mathrm{C}$ for $1 \mathrm{~h}$. Freshly prepared DAB was added for color development. The stained sections were analysed under a microscope.

IF staining of tissue sections by anti-FR Abs was performed to compare tumor-binding specificity and staining quality with the NCs-FA nanoprobes. Briefly, after blocking in serum, tissue sections were incubated at $4{ }^{\circ} \mathrm{C}$ overnight with a 1:300 dilution of mouse anti-FR protein monoclonal antibody (Abcam). The bound antibody was detected by incubating the tissues with a 1:1,000 dilution of goat anti mouse IgG-Alexa Fluor 568 at $37{ }^{\circ} \mathrm{C}$ for $1 \mathrm{~h}$. The stained sections were analysed under a microscope.

\section{Results}

\section{Synthesis and characterization of the NCs-FA fluorescent peroxidase mimetics nanoprobes}

In this study, Au NCs were covalently conjugated with FA ligands to enhance the targeting capability of the fluorescent peroxidase mimetics nanoprobes to FR over-expressing tumors. The prepared process was described in Figure 1a. First, the BSA-stabilized Au NCs was synthesized according to our previous report.[41] Second, FA was conjugated onto BSA through the EDC/NHS coupling chemistry. The size and morphology of the prepared NCs-FA nanoprobes were characterized by high resolution transmission electron microscopy (HRTEM) image. The results showed that the NCs-FA nanoprobes had a spherical shape and good dispersibility with size less than $2.0 \mathrm{~nm}$ (Figure 1b). In addition, the zeta potential of the Au NCs and NCs-FA nanoprobes were about $-20 \mathrm{mV}$ and $-38 \mathrm{mV}$ indicating good colloidal stability (date not showing). The UV-Vis absorption and fluorescent emission spectra of the NCs and NCs-FA in aqueous solution are shown in Figure 1c. The surface plasmon resonance absorption peak of the NCs and NCs-FA was not observed, which was closely related to the properties of NCs. The absorption spectrum of NCs-FA displayed the characteristic absorption peaks of the FA (365 nm), indicating the successful conjugation of FA with Au NCs. Conjugates was confirmed by ${ }^{1} \mathrm{H}$ NMR spectra ulteriorly (Supplementary Material: Figure S1). The content of FA in BSA-stabilized Au NCs was 3\% (w/w). After conjugation of FA, the fluorescent intensity of BSA-Au NCs was not changed obviously. The quantum yields of NCs and NCs-FA were $6.0 \%$ and $5.2 \%$, respectively, which permits quantification of nucleus targeting materials at single particle sensitivity. The photostability of the NCs-FA was great when compared to traditional organic fluorophores (date not showing), and the intense emission of the NCs-FA was also located in the NIR emission region $(655 \mathrm{~nm})$ for excitation at a short wavelength, which can serve as excellent bioimaging contrast agents for imaging in vitro and in vivo due to its deep penetration in tissues. (a)

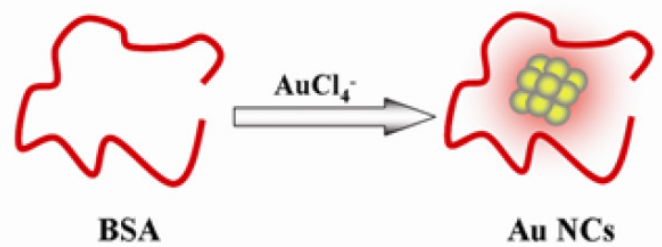

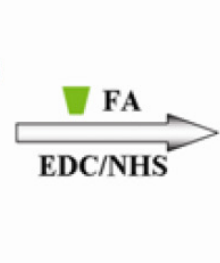

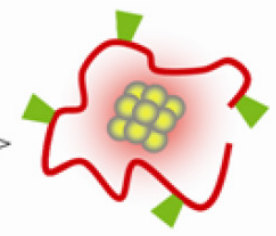

Au NCs-FA
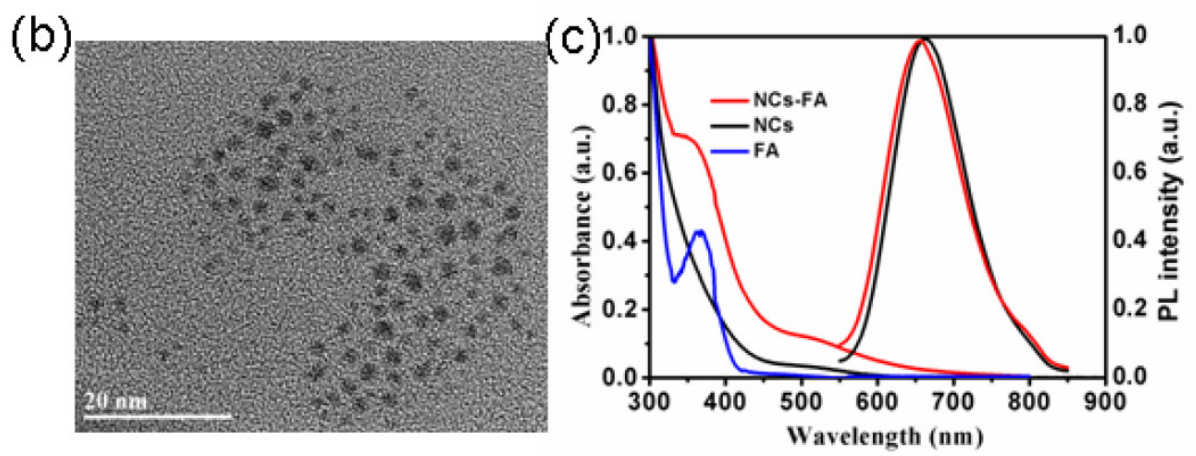

Figure I. (a) Schematic illustration of the prepared process of NCs-FA nanoprobes. (b) The HRTEM image of NCs-FA. (c) The UV-Vis absorption and fluorescent spectra. 
The colloidal stability of the as-prepared NCs and NCs-FA nanoprobes was carefully investigated under the different conditions, including buffer solutions with different $\mathrm{pH}$, salt solution with different concentrations, and different photo-irradiation time. The results showed that the fluorescence intensity of the NCs and NCs-FA nanoprobes were not obviously changed. Moreover, storage of the NCs and NCs-FA nanoprobes solution at room temperature under ambient condition for three months did not significantly change their luminescent intensity or cause precipitation, indicating its excellent stability (Supplementary Material: Figure S2). The reasons may attribute to the albumin coating layer, which was a very soluble and stable protein in different conditions. In addition, in vitro and in vivo toxicity studies showed the low toxicity of the new nanoprobes on cells or living subjects (Supplementary Material: Figure S3, S4).

\section{Enzyme mimetic activity of the NCs-FA nanoprobes}

To investigate the peroxidase-like activity of the NCs-FA nanoprobes, the catalysis of peroxidase sub- strate was tested in the presence of $\mathrm{H}_{2} \mathrm{O}_{2}$. As shown in Figure 2a-b, the NCs-FA nanoprobes catalyses the oxidation of peroxidase substrates including o-phenylenediamine (OPD), 3-Amino-9-ethylcarbazole (AEC), 5-Aminosalicylic acid (5ASA), di-azo-aminobenzene (DAB) and 3,3,5,5-tetramethylbenzidine (TMB) to give an orange color, red color, brown color, brown color and blue color, respectively, which is the same as HRP, confirming that the NCs-FA nanoprobes have peroxidase activity towards typical peroxidase substrates. In order to characterize the enzyme mimetic activities of the NCs and NCs-FA nanoprobes, a peroxidase substrate, $\mathrm{TMB}$, has been used. The catalytic activity of the NCs and NCs-FA nanoprobes was not obviously changed under different conditions, including salt solution with different concentrations, and different photo-irradiation time (Supplementary Material: Figure S5). Thus, the NCs-FA nanoprobes possess excellent stability, which are the prerequisites when acting as nanoprobes for bioimaging.

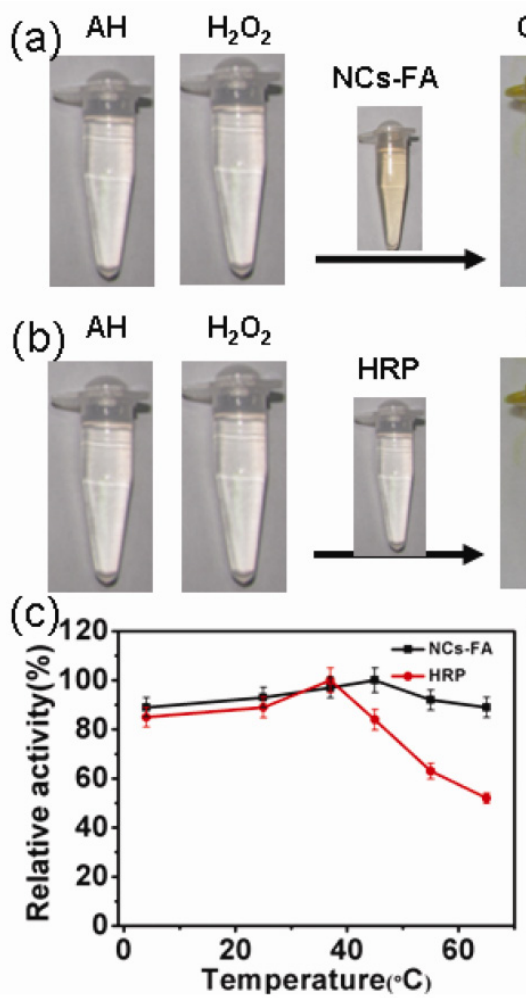

OPD AEC 5ASA DAB TMB

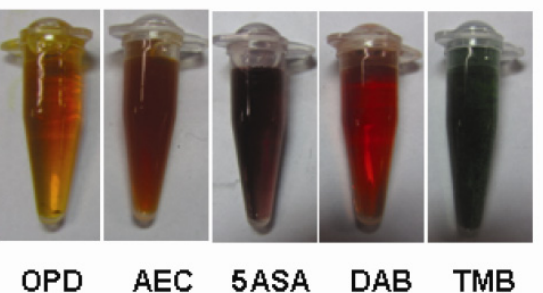

OPD AEC 5ASA DAB TMB
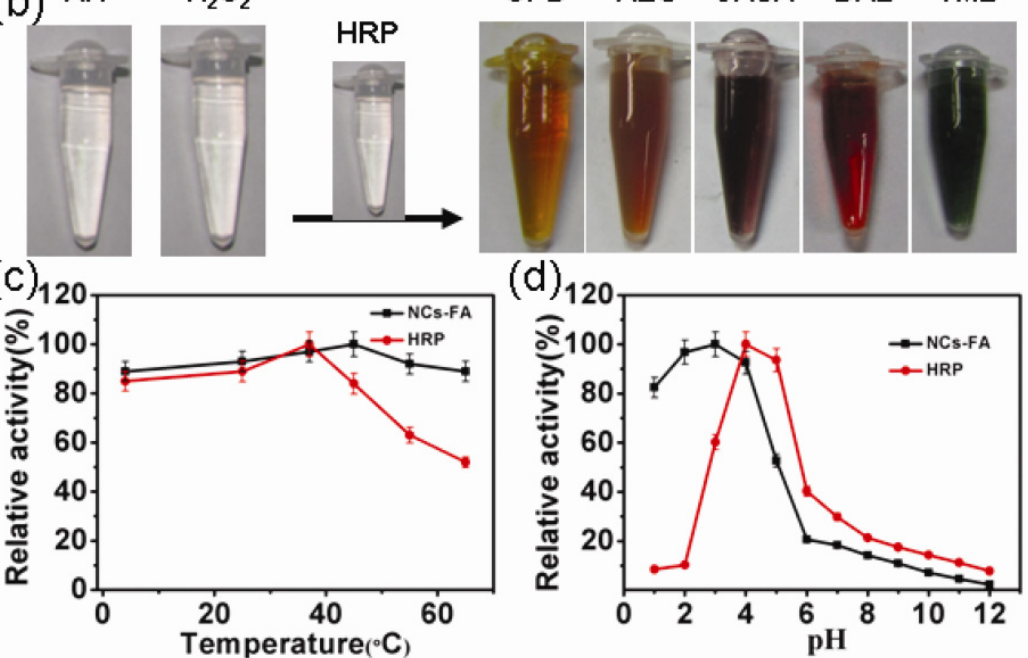

Figure 2. The NCs-FA nanoprobes (a) and HRP (b) catalyzed the oxidation of peroxidase substrates OPD, AEC, 5ASA, DAB and TMB in the presence of $\mathrm{H}_{2} \mathrm{O}_{2}$ to give the same colored products. The effects of temperatures (c) and pH values (d) on the catalytic relative activity of NCs-FA and HRP, respectively. $\mathrm{AH}$ represents the substrate, which is a hydrogen donor.

The peroxidase mimetic activity of the NCs-FA nanoprobes is, like HRP, dependent on temperature and $\mathrm{pH}$. We measured the peroxidase mimetic activity of the NCs-FA nanoprobes at different $\mathrm{pH}$ and temperature, and compared the results with the activity found in HRP. The results showed that the optimal temperature is $35^{\circ} \mathrm{C}$ and $40{ }^{\circ} \mathrm{C}$, and the optimal $\mathrm{pH}$ is 4.0 and 3.0 for HRP and the NCs-FA nano- 
probes, respectively (Figure 2c-d). Thus, we adopted $35{ }^{\circ} \mathrm{C}$ and $\mathrm{pH} 4.0$ as standard conditions for subsequent analysis of the NCs-FA nanoprobes activity. In addition, the apparent steady-state kinetic parameters were obtained, as shown in Supplementary Material: Figure S6. The NCs-FA nanoprobes-catalyzed reaction was inhibited at high $\mathrm{H}_{2} \mathrm{O}_{2}$ concentrations, as is the enzyme-catalyzed reaction. However, within the suitable range of $\mathrm{H}_{2} \mathrm{O}_{2}$ concentrations, typical Michaelis-Menten curves were observed for both NCs-FA nanoprobes and HRP. Michaelis-Menten constant $\left(\mathrm{K}_{\mathrm{m}}\right)$ and the catalytic constant $\left(\mathrm{K}_{\mathrm{cat}}\right)$ were obtained using Lineweaver-Burk plot, and the results are shown in Table 1. For natural enzymes, $\mathrm{K}_{\mathrm{m}}$ reflects the affinity between the enzyme and substrate. The lower the $K_{m}$ value is, the higher the affinity is. That means that a lower substrate concentration is required to reach the maximal activity. The apparent $\mathrm{K}_{\mathrm{m}}$ value of the NCs-FA nanoprobes with $\mathrm{H}_{2} \mathrm{O}_{2}$ as the substrate was significantly higher than that of $\mathrm{HRP}$, consistent with the observation that a higher $\mathrm{H}_{2} \mathrm{O}_{2}$ concentration was required to achieve maximal activity for the NCs-FA nanoprobes. Meanwhile, the apparent $\mathrm{K}_{\mathrm{m}}$ value of the NCs-FA nanoprobes with TMB as the substrate was lower than that of HRP. Hence, the NCs-FA nanoprobes require a lower concentration of TMB than that of HRP to reach the maximal activity. In comparison with other reported nanomaterials with peroxidase-like activities, such as $\mathrm{Fe}_{3} \mathrm{O}_{4} \mathrm{NPs}$, $\mathrm{Co}_{3} \mathrm{O}_{4}$ NPs, CGN NPs and GO-Au NCs, NCs-FA have the smallest $\mathrm{K}_{\mathrm{m}}$ values, which may be related to their small size and unique structure.[29, 42-44] The $\mathrm{K}_{\text {cat }}$ is a direct measure of the catalytic production of product under saturating substrate conditions. The greater the value of $\mathrm{K}_{\text {cat }}$, the higher is the enzymatic activity. The apparent $\mathrm{K}_{\text {cat }}$ values of the NCs-FA nanoprobes with $\mathrm{H}_{2} \mathrm{O}_{2}$ and $\mathrm{TMB}$ as the substrates were significantly higher than that of HRP. Hence, the catalytic activity of the NCs-FA nanoprobes was higher than that of HRP. In addition, the catalytic activity of the NCs-FA nanoprobes was higher than that of $\mathrm{Co}_{3} \mathrm{O}_{4} \mathrm{NPs}$ and GO-Au NCs,[42,44] and was near that of $\mathrm{Fe}_{3} \mathrm{O}_{4} \mathrm{NPs}$, CGN NPs and BSA-Au NCs.[29, 39, 43]

To understand the mechanism of the NCs-FA nanoprobes-based peroxidase mimetic reaction, the formation of $\bullet \mathrm{OH}$ during the reaction was measured when TMB was used as a peroxidase substrate. The reaction was monitored by UV-Vis absorption spectra. As shown in Supplementary Material: Figure S7, $\bullet \mathrm{OH}$ was produced during the peroxidase-like reaction in the presence of both NCs-FA nanoprobes and $\mathrm{H}_{2} \mathrm{O}_{2}$.[38] With the addition of an $\bullet \mathrm{OH}$ scavenger (ethanol), the formed $\bullet \mathrm{OH}$ disappeared and the peroxidase activity of the NCs-FA nanoprobes decreased to $15 \%$ of the original activity, indicating that the $\cdot \mathrm{OH}$ formed during the peroxidase-like reaction. Based on these results, we proposed the following possible reaction mechanism. With the addition of $\mathrm{H}_{2} \mathrm{O}_{2}$ and TMB to the NCs-FA nanoprobes reaction solution, $\mathrm{H}_{2} \mathrm{O}_{2}$ interacts with the gold core of the NCs-FA nanoprobes to generate $\cdot \mathrm{OH}$ on the surface of the gold core. The generated $\bullet \mathrm{OH}$ then oxidizes nearby TMB to form the blue color.

Table I. Comparison of the apparent Michaelis-Menten constant $\left(\mathrm{K}_{\mathrm{m}}\right)$ and catalytic constant $\left(\mathrm{K}_{\mathrm{cat}}\right)$ of the NCs-FA nanoprobes, HRP, $\mathrm{Fe}_{3} \mathrm{O}_{4} \mathrm{MNPs}, \mathrm{BSA}-\mathrm{Au} \mathrm{NCs}, \mathrm{Co}_{3} \mathrm{O}_{4} \mathrm{NPs}, \mathrm{GO}-\mathrm{Au} \mathrm{NCs}$ and CGN.

\begin{tabular}{|c|c|c|c|}
\hline Catalyst & Substrate & $\begin{array}{l}\mathrm{K}_{\mathrm{m}} \\
(\mathrm{mM})\end{array}$ & $\begin{array}{l}\mathrm{K}_{\text {cat }} \\
\left(\mathrm{s}^{-1}\right)\end{array}$ \\
\hline $\mathrm{Fe}_{3} \mathrm{O}_{4} \mathrm{MNPs}^{29}$ & TMB & 0.098 & $8.58 \times 10^{4}$ \\
\hline $\mathrm{Fe}_{3} \mathrm{O}_{4} \mathrm{MNPs}^{29}$ & $\mathrm{H}_{2} \mathrm{O}_{2}$ & 154 & $3.02 \times 10^{4}$ \\
\hline $\mathrm{Co}_{3} \mathrm{O}_{4} \mathrm{NPS}^{42}$ & TMB & 0.037 & $1.83 \times 10^{2}$ \\
\hline $\mathrm{Co}_{3} \mathrm{O}_{4} \mathrm{NPS}^{42}$ & $\mathrm{H}_{2} \mathrm{O}_{2}$ & 140.07 & $3.53 \times 10^{2}$ \\
\hline $\mathrm{CGN}^{43}$ & TMB & 0.12 & $2.48 \times 10^{4}$ \\
\hline $\mathrm{CGN}^{43}$ & $\mathrm{H}_{2} \mathrm{O}_{2}$ & 245 & $2.13 \times 10^{4}$ \\
\hline $\mathrm{BSA}-\mathrm{Au}^{39}$ & TMB & 0.00253 & $7.24 \times 10^{4}$ \\
\hline BSA-Au ${ }^{39}$ & $\mathrm{H}_{2} \mathrm{O}_{2}$ & 25.3 & $9.17 \times 10^{4}$ \\
\hline GO-AuNCs ${ }^{44}$ & TMB & 0.16 & $1.97 \times 10^{2}$ \\
\hline GO-AuNCs ${ }^{44}$ & $\mathrm{H}_{2} \mathrm{O}_{2}$ & 142.39 & $6.08 \times 10^{2}$ \\
\hline The NCs-FA nanoprobes & TMB & 0.00664 & $4.49 \times 10^{4}$ \\
\hline The NCs-FA nanoprobes & $\mathrm{H}_{2} \mathrm{O}_{2}$ & 2.46 & $8.67 \times 10^{4}$ \\
\hline HRP & TMB & 0.301 & $5.18 \times 10^{3}$ \\
\hline HRP & $\mathrm{H}_{2} \mathrm{O}_{2}$ & 0.935 & $4.05 \times 10^{3}$ \\
\hline
\end{tabular}

\section{The tumor targeting capability of the NCs-FA nanoprobes}

To explain the receptor mediated targeting capability of the NCs-FA nanoprobes to different tumors, the tumor cells were first investigated by western blot (Supplementary Material: Figure S8). The FR protein was detected in MDA-MB-231, MCF-7, SKOV3 and H460 cell lines, and the expression of FR protein in HepG2 cell lines was close to zero. To visualize the targeting cellular uptake of the NCs-FA nanoprobes, the NCs-FA nanoprobes and NCs was incubated with MCF-7 and HepG2 cells at $37^{\circ} \mathrm{C}$ for 1 $\mathrm{h}$ (Figure 3a). In the presence of NCs, almost no signal was observed from the MCF-7 cell lines. In contrast, the NCs-FA nanoprobes showed drastically enhanced fluorescence signals in MCF-7 cell lines, and showed negative signals in HepG2 cell lines. The binding of the NCs-FA nanoprobes to MCF-7 cells could be inhibited by adding an excess of unconjugated FA, further confirming that the NCs-FA nanoprobes targets tumor cells via FR. To establish the validity of the NCs-FA nanoprobes targets xenograft tumor, the NCs-FA nanoprobes and NCs were incubated with MCF-7 and HepG2 xenograft tumor section at $37{ }^{\circ} \mathrm{C}$ for $1 \mathrm{~h}$ (Figure $3 \mathrm{~b}$ ). In the presence of NCs, almost no 
signal was observed from the MCF-7 xenograft tumor. In contrast, the NCs-FA nanoprobes bound to FA-positive MCF-7 xenograft tumors, but not to FA-negative HepG2 xenograft tumor. The binding of the NCs-FA nanoprobes to FA-positive cells was saturable, and could be inhibited by adding an excess of unconjugated FA, which shown that the NCs-FA nanoprobes targets tumor cells via FR. The tumor targeting capability of the NCs-FA nanoprobes was further evaluated in tumor cell and tumor-bearing mice (Supplementary Material: Figure S9, S10). This again shows the FR-targeting of the NCs-FA nanoprobes, which have the potential to become a diagnostic tool for tumor.

\section{The NCs-FA nanoprobes for tumor diagnosis}

To establish the validity of the NCs-FA nanoprobes-based analysis method for histochemical diagnostic of tumor, we carried out the following histological staining experiments in xenograft tumors (Figure 4a). Briefly, the section from MCF-7 tumor was stained with NCs and the NCs-FA nanoprobes. After the NCs-FA nanoprobes loading and oxidation, the NCs-FA nanoprobes displayed an intensive brown peroxidase activity that visualized the positive tumor cells after adding DAB substrate and $\mathrm{H}_{2} \mathrm{O}_{2}$, and the fluorescent signal of the NCs-FA nanoprobes was also visualized in the section. In the presence of NCs, almost no signal was observed from the MCF-7 xen- ograft tumor section, verifying the feasibility of our NCs-FA nanoprobes-based tumor diagnostic method. Traditional IHC and immunofluorescence (IF) staining using anti-FR antibodies (Abs) was next performed to compare its tumor binding specificity and staining quality with our NCs-FA nanoprobes-based method in tumor tissues. The intensity and the pattern of NCs-FA nanoprobes-based staining was almost the same as that of IHC and IF staining, demonstrating the accuracy of tumor detection by the NCs-FA nanoprobes. Compared with IHC and IF, our method has the advantage of a rapid examination time, taking $1 \mathrm{~h}$, rather than the $4 \mathrm{~h}$ required for IHC and IF, which generally involves multistep incubation of primary antibody, secondary antibody or enzyme-labelled third antibody. Furthermore, NCs-FA nanoprobes-based fluorescent staining offers significant advantages over IF, including brighter fluorescent, resistance to photobleaching and optical properties, which increases the detection sensitivity. To evaluate the potential application of the NCs-FA nanoprobes as a diagnostic agent for tumors in tissue specimens, we screened other tumor tissue samples by the NCs-FA nanoprobes staining. As shown in Figure 4b, the NCs-FA nanoprobes showed strong signals in FA-positive tumor tissues (H460, MDA-MB-231, and SKOV3). This further confirmed the feasibility of our NCs-FA nanoprobes for tumor detection. (a)
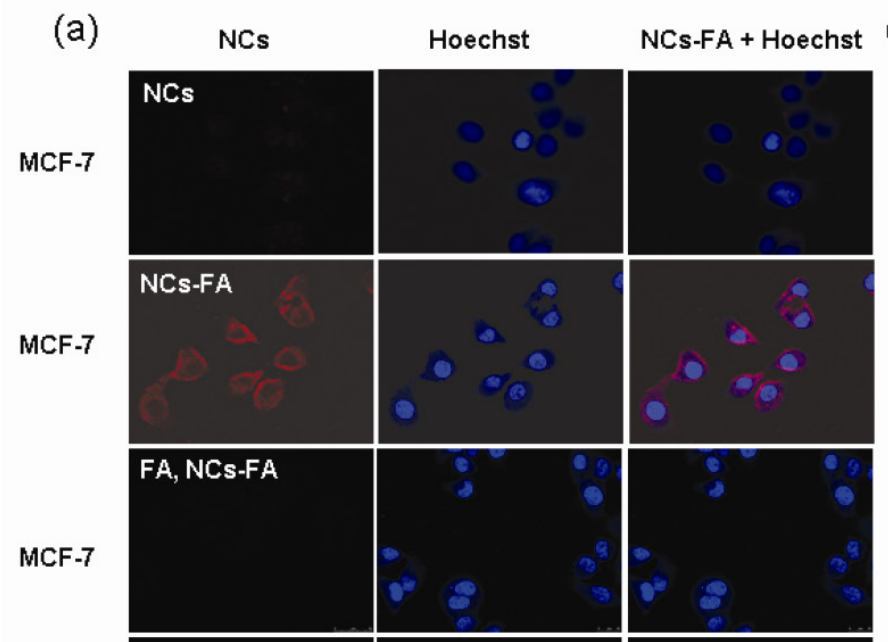

HepG2

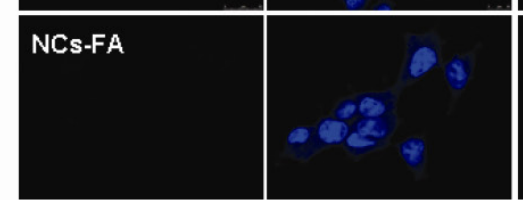

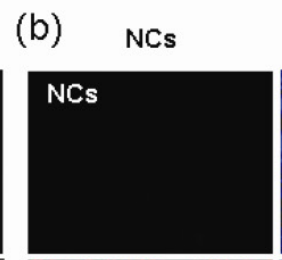
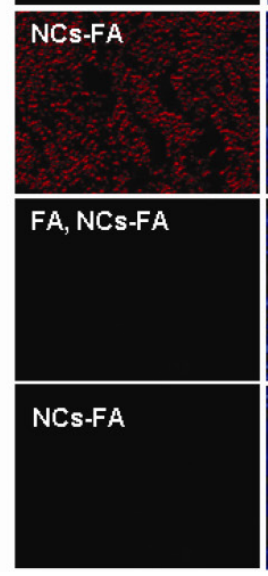

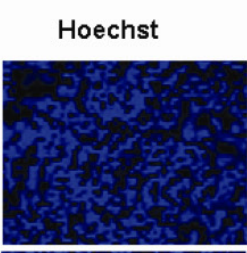

NCs-FA + Hoechst
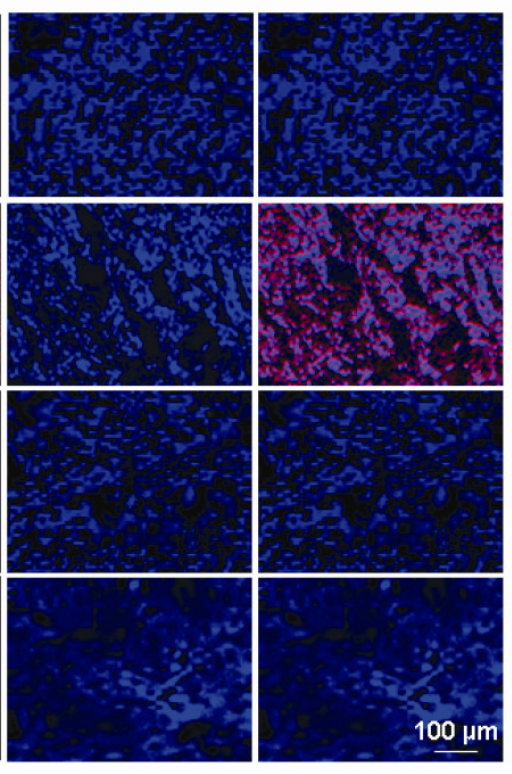

Figure 3. The NCs-FA nanoprobes bind specifically. (a) MCF-7 and HepG2 cell were stained by the NCs-FA nanoprobes (NCs) and visualized by confocal laser microscopy, respectively. (b) A MCF-7 and HepG2 tumor tissue were stained by the NCs-FA nanoprobes (NCs) and visualized by fluorescent microscopy, respectively. 
(a)
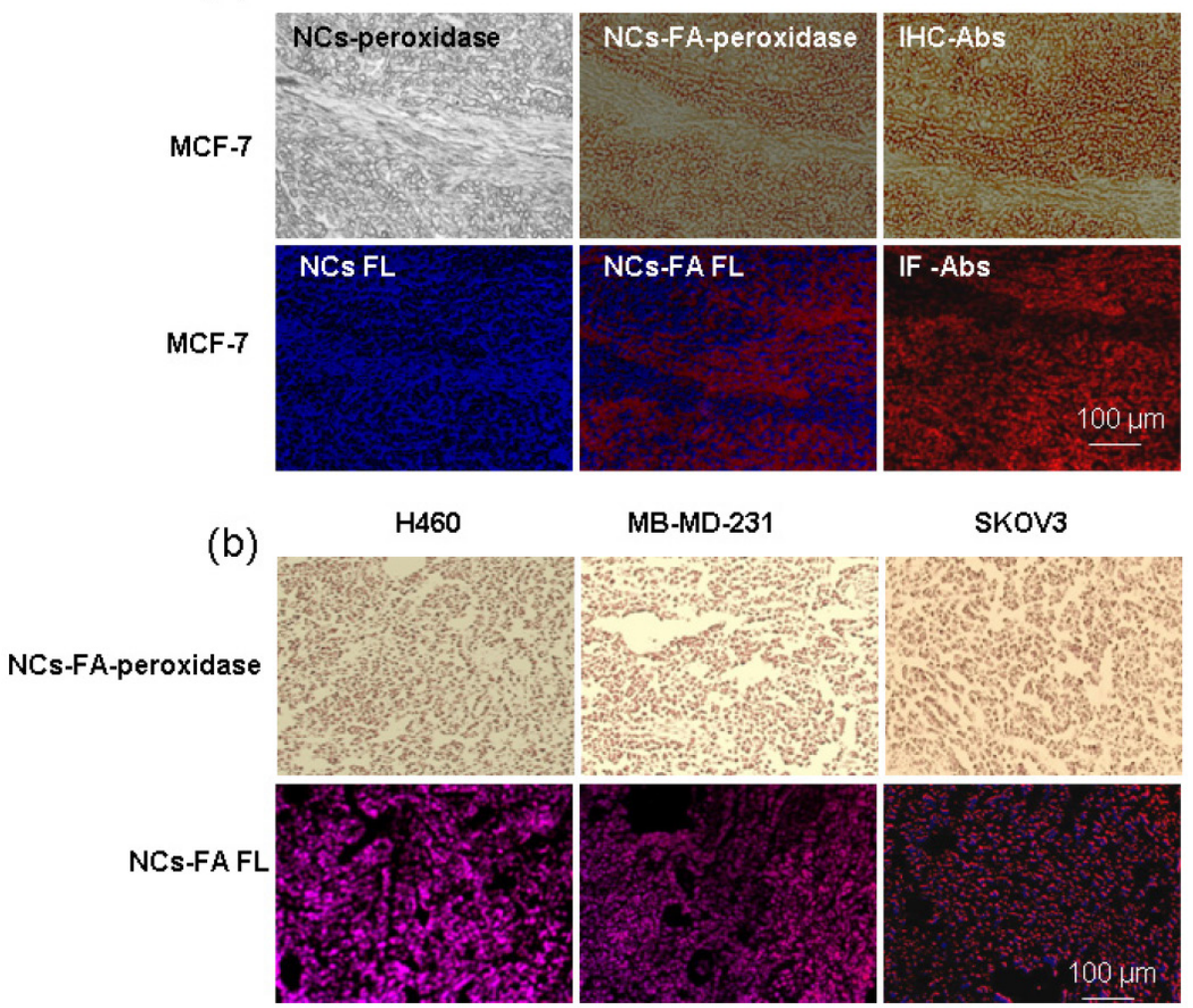

MB-MD-231

SKOV3

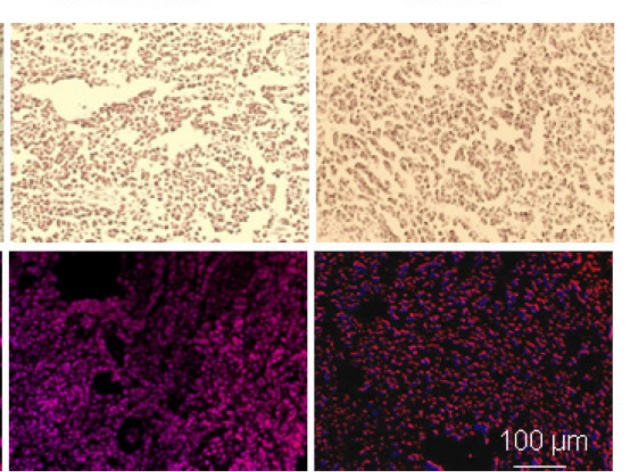

Figure 4. The NCs-FA nanoprobes for histochemical stains. (a) MCF-7 tumor tissues were stained by the NCs (NCs-FA nanoprobes and Abs), and visualized by light microscopy and fluorescent (FL) microscopy, respectively. (b) H460, MDA-MB-23 Iand SKOV3 tumor tissues were stained by the NCs-FA nanoprobes and visualized by light microscopy and fluorescent microscopy, respectively.

\section{The NCs-FA nanoprobes for molecular colocalization analysis}

The sections from MCF-7 and HepG2 tumor were stained with HRP-Abs and the NCs-FA nanoprobes, and fixated $1 \mathrm{~min}, 10 \mathrm{~min}$ and $30 \mathrm{~min}$, respectively. As shown in Supplementary Material: Figure S11, HepG-2 tumor sections showed negative staining, negative staining and positive staining, and MCF-7 tumor sections showed negative staining, positive staining and positive staining with IHC for 1 min, $10 \mathrm{~min}$ and $30 \mathrm{~min}$, respectively. The results showed that HepG-2 tumor fixated $30 \mathrm{~min}$ was false-positive and MCF-7 tumor fixated 1 min was false-negative by IHC. The results of the NCs-FA nanoprobes peroxidase staining were the same as IHC (Figure 5a). On the contrary, HepG-2 tumor sections showed negative staining, and MCF-7 tumor sections showed positive staining with the NCs-FA nanoprobes fluorescent staining for $1 \mathrm{~min}, 10 \mathrm{~min}$ and 30 min (Figure $5 b$ ). There is not any false-staining in NCs-FA nanoprobes fluorescent staining. The results showed that IHC and the NCs-FA nanoprobes peroxidase staining were not accurate, which should be supplemented by the NCs-FA nanoprobes fluorescent staining.
In order to resolve the false-positives and false-negative in IHC and the NCs-FA nanoprobes peroxidase staining, colocalization analysis was used based on the NCs-FA nanoprobes fluorescent and peroxidase staining (Figure $5 \mathrm{c}$ ). In order to study the colocalization, the bright field images of DAB were translated into fluorescent images (Figure $5 \mathrm{c}$, DAB-pseudo color). The merged fluorescent image (Figure 5c, DAB + Hoechst + NCs-FA) showed that the fluorescent staining (Figure $5 \mathrm{c}$, Hoechst + NCs-FA) similar pattern with DAB-pseudo color in tumor tissue, which also proved that the colored precipitates and fluorescent signs were generated right at the site of the NCs-FA nanoprobes-targeted tumor cells, and the oxidized colored precipitates and fluorescent signs did not diffuse away from their targets. The colocalization analysis increase the information obtained from each slide and reduce turnaround-time compared to single staining or sequential staining, and thus extend to potential clinical tests.

\section{The potential clinical application of the NCs-FA nanoprobes}

In patients diagnosed with epithelial cancer, the degree of FR over-expression is further correlated with a higher histologic grade and more advanced 
stage of the cancer, suggesting a possible need for elevated FA in more rapidly growing cancers.[45, 46] To study the potential correlation of the NCs-FA nanoprobes binding with the grade and growth pattern of cancers, bladder cancer samples (Grade 1, Grade 2, Grade 3 and Grade 4) and corresponding normal tissue samples were screened by the NCs-FA nanoprobes staining. As shown in Figure 6a, the NCs-FA nanoprobes showed a distinct staining reaction in different grades and growth patterns of bladder cancer, and the NCs-FA nanoprobes peroxidase staining similar pattern with the NCs-FA nanoprobes fluorescent staining in cancer tissue. The results demonstrated that the NCs-FA nanoprobes based staining positively correlated with differentiation, grades and growth patterns of bladder cancer. Positive cells ratio in bladder cancer was associated with a more advanced stage of the cancer, demonstrating that the NCs-FA nanoprobes has an impressive ability to discriminate tumor cells from normal cells, and thus ex- tend to potential clinical tests in cancer diagnosis (Figure 6b).

Analysis of the tumor boundary could play an important role in increasing the understanding of tumor relationships with normal structures for surgical resection planning and risk assessment for local recurrence in sarcoma. We screened clinical liver cancer tissue samples, para-carcinoma liver cancer tissue samples and corresponding normal tissue samples by the NCs-FA nanoprobes staining (Figure 6c). In cancer tissues, the NCs-FA nanoprobes strongly stained cancer cells. On the contrary, normal tissue samples consistently showed negative staining. In para-carcinoma liver cancer tissue sections, a clear distinction was seen between cancerous cells and adjacent normal cells demonstrates that the colored precipitates are generated right at the site of the NCs-FA nanoprobes-targeted cancer cells, and that the oxidized colored precipitates do not diffuse away from their targets. The method could be useful in the clinical evaluation of the cancer.

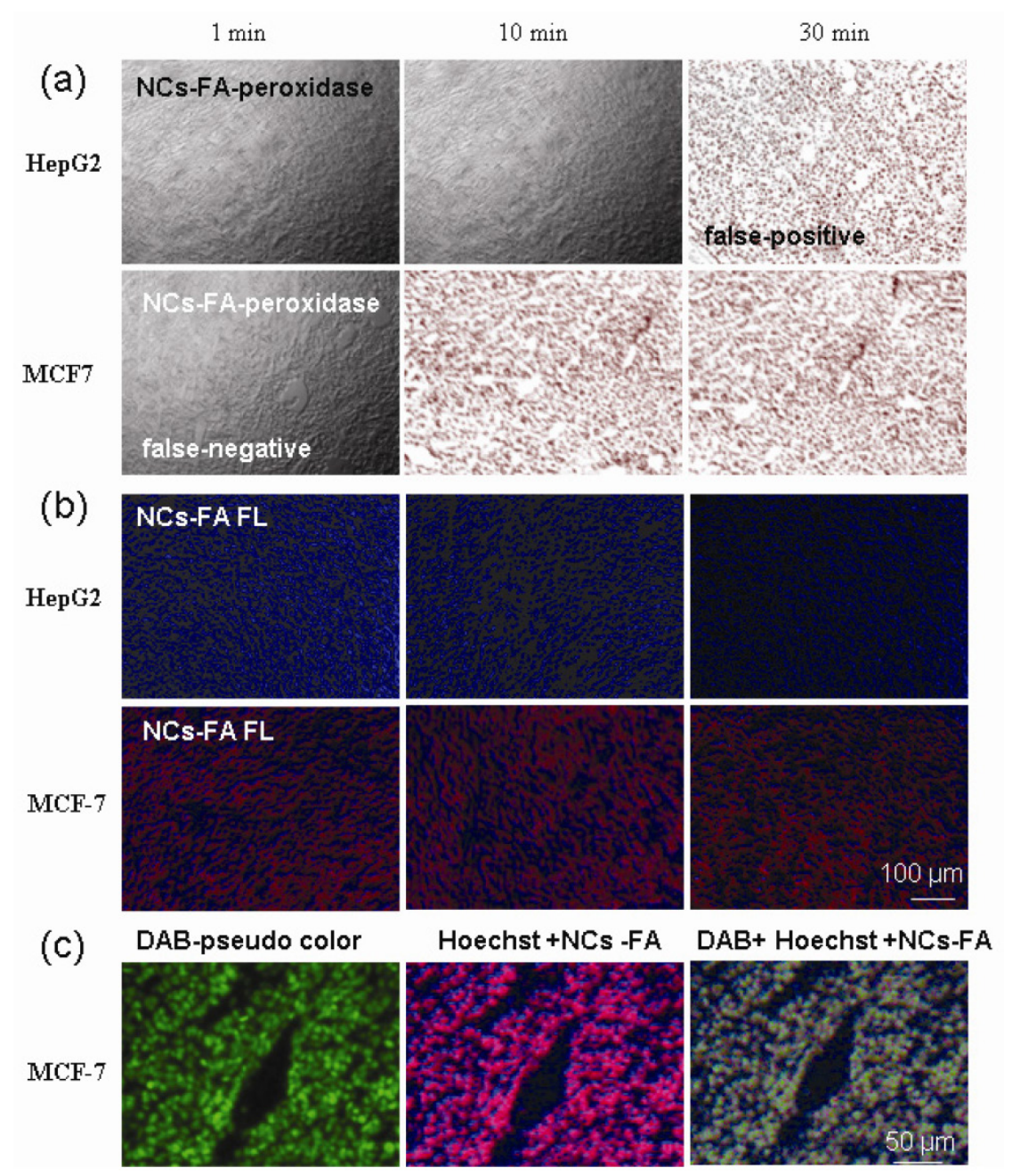

Figure 5. Colocalization Analysis. (a) The NCs-FA nanoprobes-based peroxidase staining of the sections from MCF-7 and HepG-2 tumor. (b) The NCs-FA nanoprobes-based fluorescent (FL) staining of the sections from MCF-7 and HepG-2 tumor. (c) The NCs-FA nanoprobes fluorescent staining colocalized with the NCs-FA nanoprobes peroxidase staining in tumor tissues. Abs, antibodies. 

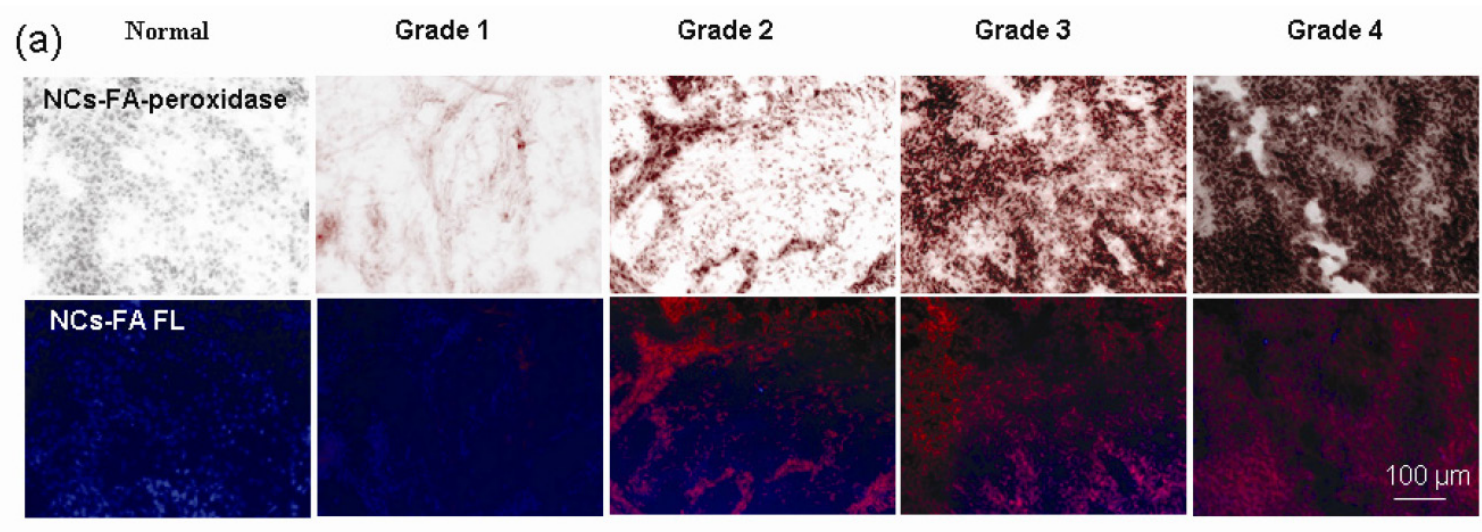

(b)

(c)
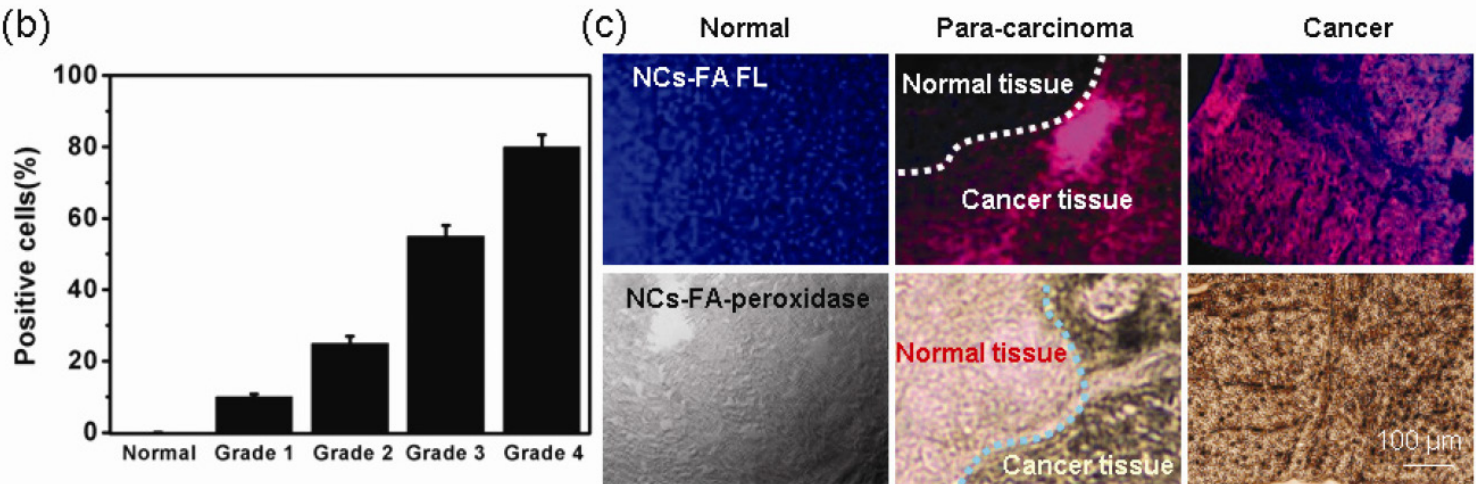

Figure 6. (a) The NCs-FA nanoprobes staining of a bladder cancer array (Normal, Grade I, Grade 2, Grade 3 and Grade 4). (b) Positive cells ratio in bladder cancer is associated with a more advanced stage of the disease. The data were represented as mean \pm standard deviation, $n=5$ per group. (c) The clinical liver cancer samples, para-carcinoma liver cancer tissue samples and corresponding normal tissue samples were stained by the NCs-FA nanoprobes.

\section{Conclusions}

In conclusion, we integrated the feature of the NCs-FA nanoprobes with NIR fluorescent imaging and peroxidase mimetics to develop a rapid, accurate, low-cost and universal analysis method for diagnosis of cancer. FA was covalently conjugated to enhance the targeting capability of the nanoprobes to FR over-expressing tumors. By using a one-step incubation of one reagent in our NCs-FA nanoprobes-based colocalization staining method, the cancer can be diagnose quickly based on microscopic imaging with bright field and fluorescent images in the same section, simultaneously, and the two images are mutually complementary. The method develops a completely custom application that takes advantage of the HRP mimetic and the fluorescent properties of the NCs-FA nanoprobes. The devised method is performed at super precision with free of false-positive and false-negative results, and higher speed than that of traditional IHC and IF. By examining different tumors and clinical samples, we verify that the NCs-FA nanoprobes could distinguish cancerous cells from normal cells. This study suggests that the NCs-FA nanoprobes have the potential to become a diagnostic tool for cancer imaging and rapid, low-cost and universal assessment of cell cancerization.

\section{Supplementary Material}

Fig.S1 - S11. http:/ / www.thno.org/v04p0142s1.pdf

\section{Acknowledgments}

The authors gratefully acknowledge support for this research from National Basic Research Program of China (973 program \#2011CB933600), the National Natural Science Foundation of China (Grant No. 81071249, 81171446, 81301272 and 20905050), Guangdong Key Laboratory of Nanomedicine, Guangdong Innovation Team of Low-cost Healthcare, and Shenzhen (CXB201005250029A, JC201104220242A, JCYJ20130401170306862, JC201005260247A, JC201005 270326A). We are grateful to Dr. Xiaojuan Sun for providing us with the clinical tumor and normal tissues.

\section{Abbreviations}

NIR, near infrared; QDs, quantum dots; Au NCs, gold nanoclusters; BSA, bovine serum albumin; IHC, immunohistochemistry; HRP, horseradish peroxidase; NPs, nanoparticles; FA, folic acid; FR, folate receptor; OPD, o-Phenylenediamine; TMB, 3,3',5,5'-Tetramethylbenzidine; DAB, 3,3'-Diaminobenzidine; 5-ASA, 5-Aminosalicylic acid; AEC, 
3-Amino-9-ethylcarbazole; PBS, phosphate-buffered saline; FBS, fetal bovine serum; DMEM, Dulbecco's Modified Eagle's Medium; HRTEM, high resolution transmission electron microscopy; MTT, methyl thiazolyl tetrazoliym; Abs, antibodies; IF, immunofluorescence; FL, fluorescent.

\section{Competing Interests}

The authors have declared that no competing interest exists.

\section{References}

1. Hu S, Arellano M, Bootheung P, Wang J, Zhou H, Jian J, et al. Salivary proteomics for oral cancer biomarker discovery. Clin Cancer Res. 2008; 14: 6246-52.

2. Ma C, Xia K, Chen H, Zeng W, Han R, Tang J. Label-free highly sensitive eetection of telomerase activity in cancer aell by ahemiluminescence imaging. Mol Cell Probes 2012; 26: 212-4.

3. Singh SK, Hawkins C, Clarke ID, Squire JA, Bayani J, Hide T, et al. Identification of human brain tumour initiating cells. Nature 2004, 432: 396-401.

4. Schamhart D, Swinnen J, Kurth KH, Westerhof A, Kuster R, Borchers H, et al. Numeric definition of the clinical performance of the nested reverse transcription-PCR for detection of hematogenous epithelial cells and correction for specific mRNA of non-target cell Origin as evaluated for prostate cancer cells. Clin Chem. 2003; 49: 1458-66.

5. Phillips JA, Xu Y, Xia Z, Fan ZH, Tan WH. Enrichment of cancer cells using aptamers immobilized on a microfluidic channel. Anal Chem. 2009; 81: 1033-9.

6. Baker, M. Whole-animal imaging: the whole picture. Nature 2010; 463: 977-80

7. Weissleder R, Pittet MJ. Imaging in the era of molecular oncology. Nature 2008; 452: 580-9.

8. Goldenberg DM, Chang CH, Rossi EA, McBride JW, Sharkey RM. Pretargeted molecular imaging and radioimmunotherapy. Theranostics 2012; 2: 523-40.

9. Niu G, Chen X. Molecular imaging with activatable reporter systems. Theranostics 2012; 2: 413-23.

10. Ahn BC. Sodium iodide symporter for nuclear molecular imaging and gene therapy: from bedside to bench and back. Theranostics 2012; 2: 392-402.

11. Sheng Z, Song L, Zheng J, Hu D, He M, Zheng M, et al. Protein-assisted fabrication of nano-reduced graphene oxide for combined in vivo photoacoustic imaging and photothermal therapy. Biomaterials 2013; 34: 5236-43.

12. Zheng CF, Zheng MB, Gong P, Jia DX, Zhang PF, Shi BH, et al. Indocyanine green-loaded biodegradable tumor targeting nanoprobes for in Vitro and in Vivo imaging. Biomaterials 2012; 33: 5603-9.

13. Kobayashi H, Ogawa M, Alford R, Choyke PL, Urano Y. New strategies for fluorescent probe design in medical diagnostic imaging. Chem Rev. 2010; 110: 2620-40.

14. Hu D, Zhang P, Gong P, Lian S, Lu Y, Gao D, et al. A fast synthesis of near-infrared emitting CdTe/CdSe quantum dots with small hydrodynamic diameter for in Vivo imaging probes. Nanoscale 2011; 3: 4724-32.

15. Kirchner C, Liedl T, Kudera S, Pellegrino T, Munoz Javier A, Gaub HE, et al. Cytotoxicity of colloidal CdSe and CdSe/ZnS nanoparticles. Nano Lett. 2005; 5: $331-8$

16. Hardman RA. Toxicologic review of quantum dots: toxicity depends on physicochemical and environmental factors. Environ. Health Perspect. 2006; 114: $165-72$.

17. Tang Z, Xu B, Wu B, Germann MW, Wang G. Synthesis and structural determination of multidentate 2,3-dithiol-stabilized Au clusters. J Am Chem Soc. 2010; 132: 3367-74

18. Xie J, Zheng Y, Ying JY. Protein-directed synthesis of highly fluorescent gold nanoclusters. J Am Chem Soc. 2009; 131: 888-9.

19. Chen H, Li S, Li B, Ren X, Mahounga DM, Cui S, et al. Folate-modified gold nanoclusters as near-infrared fluorescent probes for tumor imaging and therapy. Nanoscale 2012; 4: 6050-64

20. Hu DH, Sheng ZH, Gong P, Zhang PF, Cai LT. Highly selective fluorescent sensors for $\mathrm{Hg}^{2+}$ based on bovine serum albumin-capped $\mathrm{Au}$ nanoclusters. Analyst 2010; 135: 1411-6.

21. Xie J, Zheng $Y$, Ying JY. Highly selective and ultrasensitive detection of $\mathrm{Hg}^{2+}$ based on fluorescence quenching of $\mathrm{Au}$ nanoclusters by $\mathrm{Hg}^{2+}-\mathrm{Au}^{+}$interactions. Chem Commun. 2010; 46: 961-3.

22. Retnakumari A, Setua S, Menon D, Ravindran P, Muhammed H, Pradeep T, et al. Molecular-receptor-specific, non-toxic, near-infrared-emitting Au cluster-protein nanoconjugates for targeted cancer imaging. Nanotechnology 2010; 21: 055103 .

23. Wu X, He X, Wang K, Xie C, Zhou B, Qing Z. Ultrasmall near-infrared gold nanoclusters for tumor fluorescence imaging in Vivo. Nanoscale 2010; 2: 2244-9.

24. Leong AS, Zhuang Z. The changing role of pathology in breast cancer diagnosis and treatment. Pathobiology 2011; 78: 99-114.
25. Kumar $\mathrm{Y}$, Bhatia A, Minz RW. Antinuclear antibodies and their detection methods in diagnosis of connective tissue diseases: a journey revisited. Diagn Pathol. 2009; 4: 1-10.

26. Ramos-Vara J. A. Technical aspects of immunohistochemistry. Vet Pathol. 2005; 42: 405-26.

27. van der Loos CM. Chromogens in multiple immunohistochemical staining used for visual assessment and spectral imaging: the colorful future. The J Histotechnol. 2010, 33: 31-40.

28. Gown AM. Current issues in ER and HER2 testing by IHC in breast cancer. Mod Pathol. 2008; 21: S8-15.

29. Gao L, Zhuang J, Nie L, Zhang J, Zhang Y, Gu N, et al. Intrinsic peroxidase-like activity of ferromagnetic nanoparticles. Nat Nanotechnol. 2007; 2: 577-83.

30. Song Y, Wang X, Zhao C, Ou K, Ren J, Qu X. Label-free colorimetric detection of single nucleotide polymorphism by using single-walled carbon nanotube intrinsic peroxidase-like activity. Chem Eur J. 2010; 16: 3617-21.

31. Zhang K, Cutler JI, Zhang J, Zheng D, Auyeung E, Mirkin CA. Nanopod formation through gold nanoparticle templated and catalyzed crosslinking of polymers bearing pendant propargyl ethers. J Am Chem Soc. 2010; 132: 15151-3.

32. Narayanan R, El-Sayed MA. Changing catalytic activity during colloidal platinum nanocatalysis due to shape changes: electron transfer reaction. J Am Chem Soc. 2004; 126: 7194-5.

33. Wang CI, Chen WT, Chang HT. Enzyme mimics of Au/Ag nanoparticles for fluorescent detection of acetylcholine. Anal Chem. 2012; 84: 9706-12.

34. Song Y, Qu K, Zhao C, Ren J, Qu X. Graphene oxide: intrinsic peroxidase catalytic activity and its application to glucose detection. Adv Mater. 2010; 22: 2206-10

35. Zhai D, Liu B, Shi Y, Pan L, Wang YLi, W Zhang, et al. Highly sensitive glucose sensor based on $\mathrm{Pt}$ nanoparticle/polyaniline hydrogel heterostructures. ACS Nano 2013; 7: 3540-6.

36. Asati A, Santra S, Kaittanis C, Nath S, Perez JM. Oxidase-like activity of polymer-coated cerium oxide nanoparticles. Angew Chem Int Ed. 2009; 48: 2308-12

37. Chen Z, Yin J, Zhou Y, Zhang Y, Song L, Song M, et al. Dual enzyme-like activities of iron oxide nanoparticles and their implication for diminishing cytotoxicity. ACS Nano 2012; 6: 4001-12.

38. Fan K, Cao C, Pan Y, Lu D, Yang D, Feng J, et al. Magnetoferritin nanoparticles for targeting and visualizing tumour tissues. Nat Nanotechnol. 2012; 7: 459-64.

39. Wang XX, Wu Q, Shan Z, Huang QM. BSA-stabilized Au clusters as peroxidase mimetics for use in xanthine detection. Biosens Bioelectron. 2011; 26: 3614-9.

40. Tao Y, Lin Y, Ren J, Qu X. A dual fluorometric and colorimetric sensor for dopamine based on BSA-stabilized Au nanoclusters. Biosens. Bioelectron. 2013, 42: 41-46.

41. Hu DH, Sheng ZH, Zhang PF, Yang DZ, Liu SH, Gong P, et al. Hybrid gold-gadolinium nanoclusters for tumor targeted NIRF/CT/MRI triple-modal imaging in Vivo. Nanoscale 2013; 5: 1624-8.

42. Mu J, Wang Y, Zhao M, Zhang L. Intrinsic peroxidase-like activity and catalase-like activity of $\mathrm{CO}_{3} \mathrm{O}_{4}$ nanoparticles. Chem Commun. 2012; 48: 2540-2.

43. Adhikamsetty R, Gollapalli N, Jonnalagadda S. Complexation kinetics of $\mathrm{Fe}^{2+}$ with 1,10-phenanthroline forming ferroin in acidic solutions. Int J Chem Kinet. 2008; 40: 515-23

44. Tao Y, Lin Y, Huang Z, Ren J, Qu X. Incorporating graphene oxide and gold nanoclusters: a synergistic catalyst with surprisingly high peroxidase-like activity over a broad $\mathrm{pH}$ range and its application for cancer cell detection. Adv Mater. 2013; 25: 2594-9.

45. Toffoli G, Cernigoi C, Russo A, Gallo A, Bagnoli M, Boiocchi M. Overexpression of folate binding protein in ovarian cancers. Int J Cancer. 1997; 74: 193-8.

46. Lu YJ, Low PS. Folate-mediated delivery of macromolecular anticancer therapeutic agents. Adv. Drug Delivery Rev. 2012; 64: 342-52. 